\title{
Effectiveness of multidisciplinary intervention in obese women with binge eating disorder
}

\begin{abstract}
Objective: Identify if binge eating disorder (BED) interfere in anthropometric (weight and waist circumference-WC), clinical and metabolic parameters compared to Non Binge Eating Disorder (NBED) and if a multidisciplinary treatment improve those parameters.

Methods: A 24-week of multidisciplinary intervention consisting of an either behavioral (hypocaloric diet and exercise stimulus) and cognitive behavioral therapy (CBT). Anthropometry was performed monthly. CBT performed weekly in eight sessions. Biochemical, inflammatory biomarkers (Leptin and adiponectin), body composition and binge eating score (BES) were measured at baseline and 24-weeks. A $p<0.05$ was considered significant.

Results: Thirty-three women were studied, 14 NBED and 19 BED. Leptin and BES were higher in BED. After intervention, anthropometric parameters diminished in both groups, with a mean $4 \%$ weight reduction. Total cholesterol and fat mass reduced in the BED women; HDL-cholesterol and fat-free mass reduced in NBED women. Both groups maintained high hypertension prevalence. In BED, there was a positive correlation between leptin and BMI as well as in WC and a negative correlation among leptin and fat-free mass. After intervention there was a reduction on binge episodes: frequency and severity.
\end{abstract}

Conclusion: Multidisciplinary intervention for treating obesity improves anthropometric, clinical and metabolic parameters independently of having or not BED.

Keywords: obesity, women, binge eating disorder, anthropometry, treatment

\author{
Volume 7 Issue 2 - 2017
}

\author{
Henyse Gomes Valente da Silva,' Adriana \\ Paulino do Nascimento, ${ }^{2}$ Luis Cristovao \\ Porto, ${ }^{3}$ Annie Belo Moreira ${ }^{4}$ \\ 'Integral Medicine Department, Rio de Janeiro State University, \\ Brazil. \\ ${ }^{2}$ Roberto Alcantara Gomes Biology Institute, Rio de Janeiro \\ State University, Brazil \\ ${ }^{3}$ Roberto Alcantara Gomes Biology Institute nd Policlinica \\ Piquet Carneiro, Rio de Janeiro State University, Brazil \\ ${ }^{4}$ Applied Nutrition Department, Rio de Janeiro State University, \\ Brazil
}

Correspondence: Henyse Gomes Valente da Silva, Integral Medicine Department, Rio de Janeiro State University, Boulevard 28 de setembro 77, Rio de Janeiro, Brazil, Tel +55 21999849833,Email henyse@uol.com.br

Received: August 31, 2017 | Published: September 29, 2017

\section{Abbreviations}

$\mathrm{BCM}$, body cell mass; $\mathrm{BED}$, binge eating disorder; $\mathrm{BES}$, binge eating scalel; BIA, bioelectrical impedance analysis; BMI, body mass index; BT, behavioral therapy; CBT, cognitive-behavioral therapy; FFM, total fat-free body mass in $\mathrm{Kg}$; FM, fat body mass in $\mathrm{Kg}$; MetS, metabolic syndrome; NBED, non binge eating disorder; WC, waist circumference

\section{Introduction}

Binge eating disorder (BED) is a psychiatric disorder characterized by eating large amounts of food, not followed by inappropriate compensatory behaviors for weight control that characterize other eating disorders. In 2013, it was approved, by the American Psychiatric Association, the inclusion of this disorder in the diagnostic and statistical manual of mental disorders 5th edition (DSM-V) as its own category of eating disorder. ${ }^{1}$ There is an important relationship between this disorder and obesity, which complexity is linked to psychological and physical implications, such as a low self-esteem, weight and shape concerns, and unhealthy eating behaviors including binge eating. ${ }^{2}$

It is a relatively common disorder, in general population, with estimated lifetime prevalence around $1.4 \%,{ }^{3}$ but reaching higher levels from $2 \%$ to $3 \%{ }^{4}$ When considered obese individuals seeking treatment for weight loss in addition to BED, the proportion increases to approximately $5 \%$ to $30 \%{ }^{5}$
Adipose tissue-derived hormones are involved in the pathophysiology of eating disorders and other mental disorders. These appetite-influencing hormones, such as leptin and adiponectin, are respectively increased and reduced in obesity in individuals with or without BED, ${ }^{6}$ and linked to visceral abdominal fat that is recognized as a passive fat storehouse with high capacities of adipokines synthesis, leading to proinflammatory and insulin-resistant states. ${ }^{7}$ This metabolic pattern modification could be related to altered angiogenesis in adipose tissue and an important increase in the cardiovascular risk. $^{8}$

Planning highly individualized, multidisciplinary and steppedcare treatment seems to be the more promising strategy. It is necessary to deal with low self-esteem experiences, weak therapeutic alliance, predictable treatment dissatisfaction and early discontinuation of care. The therapeutic setting should be flexible and considering psychological and physical impairments, associated with motivation on their condition. ${ }^{9,10}$

The first goal of treatment is to achieve abstinence from binge eating, since it, per se, can leads to significant weight loss. Afterward, a sustainable weight loss is the objective. On this purpose, the education to healthier eating and life styles, and the modification of dysfunctional thoughts and habits are essentials. The most evaluated and developed intervention for BED includes behavioral (BT) and Cognitive-Behavioral Therapies (CBT). ${ }^{11,12}$ Behavioral treatments, focused on diet and lifestyle modification, and caloric restrictions should be moderate, and a normal or hypo caloric diet seems to be 
the best strategy to prevent bingeing. Any effort must be encouraged and poor compliance to diet should not be blamed. ${ }^{13}$ Physical activity acts on fat consumption and diminish excessive food intake. It helps maintaining goals reached with diet, and it improves mood and general health, lowering clinical comorbidities of BED.${ }^{14}$ Moreover, behavior weight loss therapy has a higher immediate binge reduction at shorttime observations than cognitive behavioral therapy. Unfortunately, evidences about effects on weight reduction are still limited and of unclear clinical significance. ${ }^{15}$ On the other hand, the CBT has been shown to be more effective than weight loss therapy alone, over the long-term therapy. Their success rate varies from $50-60 \%$. This can be explained by dual process theories that suggest attention bias can influence behavior and explain improve outcomes for people who binge eating. ${ }^{16}$

The aim of this study was to identify if there is any difference in anthropometric, biochemical and appetite-influencing hormone profile, comparing obese individuals with or without BED, and to evaluate if there is any clinical, inflammatory or metabolic profile change after a 24 week-multidisciplinary intervention.

\section{Materials and methods}

This was a longitudinal study involving 33 women who sought weight loss and agreed to be evaluated monthly according to our multidisciplinary therapy. It was conducted between 2013-2014, in outpatients' Rio de Janeiro State University - Policlinica Piquet Carneiro.

Inclusion criteria were obesity (body mass index $\geq 30 \mathrm{~kg} / \mathrm{m}^{2}$ ), having binge eating disorder (BED) or not (NBED), aged 18 to 60, pre-or post-menopause. Pre-menopausal participants were evaluated in the first ten days of menstrual cycle. Exclusion criteria were pregnancy, nursing mothers, consumptive or malignant diseases (cancer, AIDS, liver and degenerative diseases), mental health problems and use of anti-inflammatory or corticosteroid drugs.

The assessment of obesity followed the recommendation of the World Health Organization and considered values of BMI (weight in kilograms divided by the square of the height in meters) $\geq 30-34.9=$ grade $1 ; \geq 35-39.9=$ grade $2, \geq 40 \mathrm{Kg} / \mathrm{m}^{2}$ grade $3 .{ }^{17}$

Diagnose of the binge eating disorder was made by a mental health specialist based on standardized clinical interviews, according to DMS-5, that included: binge eating at least once a week for at least three months, and dichotomized as NBED- not having or BED- having binge eating disorder. ${ }^{1}$ Individuals with any other mental health disease were excluded from the study. The BED was classified in moderate or severe according to a Binge Eating Scale (BES) translated into Portuguese and validated for the Brazilian population. It is a 16-item self-reported questionnaire, designed specifically to identify behavioral and cognitive characteristics of binge eating in obese individuals. ${ }^{18}$ Each item presents three or four differently weighted statements, with a final score varying from 0-46. The classification depends on the total score: non-bingers (scoring 17 and less), moderate bingers (scoring between 18 and 26), and severe binger's eaters (scoring 27 and above).

Data were obtained at baseline and 24weeks after the intervention and included: epidemiological, anthropometry (height, weight and waist circumference - WC), bioelectrical impedance analysis (BIA) for body composition, arterial blood pressure, laboratorial parameters and binge score.
Women were stratified per physical activity, smoking habits, alcohol consumption hypertension, dyslipidemia and Metabolic Syndrome (MetS), that was classified according to NCEP-ATP III 2005, including: WC, hypertension, triglycerides, HDL-cholesterol and glycemic level. ${ }^{19}$

Activities lower than $60 \mathrm{~min} /$ weekly, characterized a sedentary behavior. ${ }^{14}$ Classification of smoking and alcohol habits were selfassumed. Characterization of dyslipidemia and hypertension followed the Brazilian Society of Cardiology recommendation. ${ }^{20}$

A biodynamics $310 \mathrm{e}$ analyzer was used to perform the BIA, performed in the morning period. The electrodes were placed in the standard tetra polar position of the right side of the patient. The software Fluids \&Nutrition (version 4.0) was used to estimate Total Fat-Free Body Mass in $\mathrm{Kg}$ (FFM), Fat Body Mass in $\mathrm{Kg}$ (FM) and Body Cell Mass (BCM) that included muscle (60\%), organs $(20 \%)$, and circulating cells and tissues. ${ }^{21}$

Blood samples were collected after 12 hours of overnight fasting, at the beginning and at the end of the 24-week intervention, and included serum glucose levels, lipids and adipose tissue-derived hormones: Leptin and Adiponectin. Women with normal laboratory values were those whose leptin=3,877-77,273 $\mathrm{pg} / \mathrm{mL}$ and adiponectin $=5,000$ $25,000 \mathrm{ng} / \mathrm{mL}$. Lipids and glucose level were evaluated according to NCEP-ATP III recommendation. ${ }^{19}$

Analysis performed by an automated method, using commercial kits (Abbot Architect $\mathrm{c} 8000{ }^{\circledR}$, Abbott Park, IL, USA) was used to evaluate serum triglyceride, total cholesterol and HDL-cholesterol. The LDL-cholesterol level was calculated using the Friedewald formula. ${ }^{22}$

The multidisciplinary intervention included BT, evaluated every month for both groups, and a weekly CBT, only for BED group. Independently of having or not BED the multidisciplinary approach had the same intensity intervention. None BED individuals required medication. The CBT included self-monitoring, goal setting, stimulus control and discussion over problems with adherence to recommendations on diet and exercise and was performed in eight moments.

The behavioral therapy included orientation for exercising more than $150 \mathrm{~min}$ weekly and a dietary plan for weight reduction based on recommended daily allowances intake for obesity. Caloric intake was reduced by $500 \mathrm{kcal}$, setting between $1,200-2,000 \mathrm{kcal} / \mathrm{day}$. Macronutrients recommendation were: carbohydrates: $60 \%$, proteins: 1-to-1.2g/weight kilogram, and lipids to complete calories Sucrose additives were removed from the diet. Fruits and vegetables were included three to four times a day. Meals were recommended to be consumed five or six moments a day to prevent excessive hunger, and a food replacement list was offered. ${ }^{12,23}$

The primary end was adhering to therapy after the 24-week intervention. Secondary ones included weight, body composition, arterial blood pressure, dyslipidemia and Adipose tissue-derived hormones improvement. The study was approved by the Research Ethics Committee of the Rio de Janeiro State University, under Protocol No 2774/2010. All participants signed the informed consent document after gathering procedures information, risks and benefits from the research.

Statistical analyses were conducted by IBM ${ }^{\circledR}$ SPSS ${ }^{\circledR}$ Statistics software version 21 . The results were presented as the mean \pm SD 
or median $\left(25^{\text {th }}-75^{\text {th }}\right.$ percentiles $)$. The normality of the variables was investigated using the Kolmogorov-Smirnov test. To assess differences in categorical variables between the groups that completed the study protocol and follow-up losses, the Chi-square test was used. To assess the intra intervention group's (BED and NBED) effect on anthropometric, clinical and laboratory measures, the paired Student's t-test or Wilcoxon Signed Ranks were used. The effect between groups was assessed by the Mann-Whitney U or Student's t-test according to the distribution of variables. Pearson or Spearman correlation test was used to assess the existence, magnitude and direction of linear association between BMI, WC, leptin and adiponectin, considering the correlation coefficients, confidence interval, and $\mathrm{p}$ value. A significance level of $\mathrm{p}<0.05$ was considered significant.

\section{Results and discussion}

One hundred and seven women were assessed for eligibility, but only 33 underwent the study as they completed the 24-week intervention. Fourteen were assigned to the NBED and 19 to the BED group. Both groups had a similar number of post-menopausal women which did not modify the statistical analysis between them. Table 1 shows the characteristics of patients who completed the study.

Table I Baseline clinical, anthropometric and laboratory characteristics differences according to according to having (BED) or not binge eating disorder (NBED). Results are expressed as mean \pm SD or prevalence, total (percent)

\begin{tabular}{|c|c|c|c|}
\hline \multirow{2}{*}{ Characteristics } & \multirow{2}{*}{$\begin{array}{l}\text { NBED } \\
(n=\mid 4)\end{array}$} & \multirow{2}{*}{$\begin{array}{l}\text { BED } \\
(n=19)\end{array}$} & \multirow{2}{*}{$P$ value } \\
\hline & & & \\
\hline \multicolumn{4}{|l|}{ General } \\
\hline Age (years) & $43.1 \pm 12.5$ & $46.1 \pm 11.6$ & 0.497 \\
\hline Dislipidemia. n (\%) & $5(35.7)$ & $6(31.6)$ & 0.547 \\
\hline Hypertension. n (\%) & $7(50)$ & II (57.6) & 0.461 \\
\hline Smokers. n (\%) & $0(0)$ & $2(10.5)$ & 0.324 \\
\hline Alcohol user. n (\%) & $2(14.3)$ & $3(15.8)$ & 0.649 \\
\hline Sedentary. n (\%) & $13(92.8)$ & 15 (78.9) & 0.278 \\
\hline MetS.n (\%) & $9(64.3)$ & $10(52.3)$ & 0.518 \\
\hline Obesity Grade/Level.n (\%) & & & 0.792 \\
\hline I & $6(42.9)$ & $4(21.1)$ & \\
\hline II & $3(21.4)$ & II (57.9) & \\
\hline III & $5 \mid(35.7)$ & $4(2 \mid .1)$ & \\
\hline BES & $10.34 \pm 9.30$ & $22.53 \pm 4.39$ & 0.02 \\
\hline \multicolumn{4}{|l|}{ Anthropometric } \\
\hline Weight (kg) & $90.5 \pm 13.4$ & $100.0 \pm 19.4$ & 0.126 \\
\hline $\mathrm{BMI}\left(\mathrm{kg} / \mathrm{m}^{2}\right)$ & $38.5 \pm 5.9$ & $39.1 \pm 5.7$ & 0.5443 \\
\hline$W C(\mathrm{~cm})$ & $106.6 \pm 11.7$ & $109.6 \pm 13.1$ & 0.5025 \\
\hline SBP $(\mathrm{mmHg})$ & $|3| .5 \pm \mid 4.0$ & $|26.5 \pm| 2 . \mid$ & 0.4371 \\
\hline $\mathrm{DBP}(\mathrm{mmHg})$ & $91.2 \pm 18.0$ & $84.6 \pm 9.0$ & 0.1726 \\
\hline Fat free Mass $(\mathrm{Kg})$ & $52.8 \pm 5.2$ & $56.5 \pm 9.1$ & 0.1814 \\
\hline Fat Mass (Kg) & $37.7 \pm 9.2$ & $43.9 \pm 11.3$ & 0.1037 \\
\hline Fat $\%$ & $4 I . I \pm 4.6$ & $43.3 \pm 3.3$ & 0.723 \\
\hline \multicolumn{4}{|l|}{ Biochemical } \\
\hline Glucose (mg/dL) & $98.0 \pm 9.5$ & $107.4 \pm 20.7$ & 0.2678 \\
\hline Triglycerides (mg/dL) & $96.5 \pm 33.1$ & $116.0 \pm 56.2$ & 0.787 \\
\hline Total cholesterol (mg/dL) & $181.6 \pm 30.8$ & $198.1 \pm 39.5$ & 0.2745 \\
\hline HDL-cholesterol (mg/dL) & $49.6 \pm 12.4$ & $48.9 \pm 7.7$ & 0.85 \\
\hline LDL-cholesterol (mg/dL) & $113.0 \pm 24.4$ & $125.2 \pm 36.0$ & 0.3634 \\
\hline Leptin (pg/ml) & $38498 \pm 21020$ & $51124 \pm 20809$ & $0.0416 *$ \\
\hline Adiponectin (ng/ml) & $5764 \pm 3693$ & $7020 \pm 3813$ & 0.2513 \\
\hline
\end{tabular}

MetS, metabolic syndrome; BMI, body mass index; WC, waist circumference; SBP, systolic blood pressure; DBP, diastolic blood pressure; HDL, high density lipoprotein; LDL, low density lipoprotein; CRP, c - reactive protein; BES, binge eating scale; *P<0.05; T, student test- parametric dates; Mann-Whitney Test: No parametric dates. 
The evaluation revealed no significant difference in baseline epidemiological, anthropometry - weight and WC, BIA, arterial blood pressure and laboratorial parameters, in both groups. Leptin was the only adipose tissue-derived hormone, statistically different, and higher, in the BED women. Based on binge eating score, there was high binge score prevalence in the BED group.

After 24weeks of intervention, both groups benefited with significant anthropometric reduction. Weight reduction varied from $1 \%$ to $20 \%$, with a mean reduction of $4 \%$, with no differences in the two groups. Respectively $74 \%$ of BED and $64 \%$ of NBED lost weight. A reduction in fat mass was only observed in the BED group; while a fat-free mass reduction was exclusively noticed in NBED women. There was no systolic or diastolic blood pressure reduction in either group, and no significant between-group differences. Both groups maintain a high prevalence of hypertension and no exercise practice changing. Smoking and alcohol consumption did not improve.

The lipids profile improved. The BED group benefited with a significant reduction in total cholesterol level and the NBED with a HDL cholesterol increase. No significant difference in LDL cholesterol, triglyceride or glucose levels was observed in either group at 24 weeks.

There was a reduction on binge episodes' frequency and severity. Around 30\% have their BES classification moving from severe to moderate binger eating after treatment. All these results are shown in Table 2.

Table 2 Anthropometric, biochemical parameters and binge eating: score Pre-and variation after 24weeks multidisciplinary intervention (delta) according to having (BED) or not binge eating disorder (NBED). Results are expressed as mean \pm SD

\begin{tabular}{|c|c|c|c|c|c|c|c|}
\hline \multirow{2}{*}{ Variables } & \multicolumn{3}{|l|}{ NBED $(n=\mid 4)$} & \multicolumn{3}{|c|}{ BED $(n=19)$} & \multirow{2}{*}{$\begin{array}{l}\text { P value Delta } \\
\text { NBED x BED }\end{array}$} \\
\hline & pre & Delta & $\begin{array}{l}\text { p value pre } x \\
\text { post }\end{array}$ & Pre & Delta & $\begin{array}{l}\text { P value pre } x \\
\text { post }\end{array}$ & \\
\hline \multicolumn{8}{|l|}{ Anthropometric } \\
\hline Weight (kg) & $90.5 \pm 13.4$ & $-3.2 \pm 5.0$ & $0.0335^{*}$ & $100.0 \pm 19.4$ & $-3.9 \pm 3.4$ & $<0.0001 *$ & 0.638 \\
\hline BMI $\left(\mathrm{kg} / \mathrm{m}^{2}\right)$ & $38.5 \pm 5.9$ & $-4.5 \pm 12.0$ & $0.0345^{*}$ & $39.1 \pm 5.7$ & $-4.1 \pm 1.3$ & $<0.000 I^{*}$ & 0.282 \\
\hline WC $(\mathrm{cm})$ & $106.6 \pm 11.7$ & $-4.7 \pm 7.2$ & $0.0320 *$ & $109.6 \pm 13.1$ & $-3.2 \pm 5.3$ & $0.0167 *$ & 0.999 \\
\hline Fat-free mass (kg) & $52.8 \pm 5.2$ & $-1.5 \pm 1.8$ & $0.0102 *$ & $56.5 \pm 9.1$ & $-0.7 \pm 2.1$ & 0.2094 & 0.272 \\
\hline Fat Mass (Kg) & $37.7 \pm 9.2$ & $-1.7 \pm 4.5$ & 0.1773 & $43.9 \pm 11.3$ & $-3.0 \pm 2.9$ & $0.0005^{*}$ & 0.342 \\
\hline Fat $\%$ & $4 I . I \pm 4.6$ & $-0.7 \pm 3.7$ & 0.4612 & $43.3 \pm 3.0$ & $-1.4 \pm 2.4$ & $0.0093 *$ & 0.544 \\
\hline \multicolumn{8}{|l|}{ Biochemical } \\
\hline Glucose (mg/dL) & $98.0 \pm 9.5$ & $+3.6 \pm 6.6$ & 0.0872 & $107.4 \pm 20.7$ & $-3.4 \pm 12.5$ & 0.8926 & 0.099 \\
\hline Triglyceride (mg/dL) & $96.5 \pm 33.1$ & $-6.4 \pm 26.1$ & 0.4370 & $116.0 \pm 56.2$ & $+1.0 \pm 46.7$ & 0.5049 & 0.647 \\
\hline T.Cholest (mg/dL) & $181.6 \pm 30.8$ & $-1.2 \pm 24.1$ & 0.8739 & $198.1 \pm 39.5$ & $-10.9 \pm 16.2$ & $0.0321 *$ & 0.157 \\
\hline HDL-cholest(mg/dL) & $49.6 \pm 12.4$ & $+8.8 \pm 11.7$ & $0.0317^{*}$ & $48.9 \pm 7.7$ & $-0.9 \pm 4.2$ & 0.4466 & $0.010 *$ \\
\hline LDL-cholest mg/dL) & I I $3.0 \pm 24.4$ & $-6.4 \pm 24.1$ & 0.4021 & $125.2 \pm 36.0$ & $-6.6 \pm 16.2$ & 0.2044 & 0.975 \\
\hline Leptin (pg/ml) & $38498 \pm 21020$ & $+7715.2 \pm 1685.2$ & 0.1189 & $\begin{array}{l}51124 \pm \\
20809\end{array}$ & - & 0.8596 & 0.11 \\
\hline Adiponectin(ng/ml) & $5764 \pm 3693$ & $+517.6 \pm 248.0$ & 0.0906 & $7020 \pm 3813$ & $\begin{array}{l}+ \\
254.3 \pm 1412.3\end{array}$ & 0.4427 & $70 \%$ \\
\hline BES & $10.34 \pm 9.30$ & NE & NE & $22.53 \pm 4.39$ & $-7.45 \pm 2.82$ & 0.045 & 0.02 \\
\hline
\end{tabular}

MetS, metabolic syndrome; BMI, body mass index; WC, waist circumference; SBP, systolic blood pressure; DBP, diastolic blood pressure; HDL, high density lipoprotein; LDL, low density lipoprotein; CRP, c - reactive protein; BES, binge eating scale; *P<0.05; T, student test- parametric dates; Mann-Whitney Test: No parametric dates. 
Before the intervention, the linear regression analysis revealed that leptin was positively correlated with BMI $(\mathrm{r}=0.65, \mathrm{p}=0.001)$, WC $(\mathrm{r}=0.67, \mathrm{p}=0.001)$ and fat percentage $(\mathrm{r}=0.74, \mathrm{p}=0.001)$ (Table 3$)$. No correlation was observed between adiponectin and anthropometric parameters.

Table 3 Correlation analyses among leptin and anthropometric parameters: Before multidisciplinary intervention

\begin{tabular}{llll}
\hline \multirow{2}{*}{$\begin{array}{l}\text { Antropometric } \\
\text { parameters }\end{array}$} & Leptin & \\
\cline { 2 - 4 } & All Patient (r) & NBED(r) & BED(r) \\
\hline BMI & $0.65^{* * *}$ & $0.75^{* *}$ & 0.41 \\
WC & $0.67^{* * *}$ & $0.76^{* *}$ & $0.60^{*}$ \\
Fat \% & $0.74^{* * *}$ & $0.78^{* *}$ & $0.5 I^{*}$ \\
\hline
\end{tabular}

BMI, body mass index; WC, waist circumference; NBED, not binge eating disorder; BED, binge eating disorder; Pearson correlation test ${ }^{*} \mathrm{p}<0.05$; ${ }^{* *} \mathrm{p}<$ $0.01 ; * * * \mathrm{p}<0.001$

In evaluating the two groups separately, the positive correlation between leptin and anthropometric markers: BMI, WC and Fat \%, was stronger in the BED group than in the obese without BED. In nonbinge eating obese women, leptin levels did not correlate with BMI.

After the intervention, leptin was still positively correlated with BMI $(r=0.58, p=0.01)$, and fat percentage $(r=0.52, p=0.02)$ in the $\mathrm{BED}$ group. The negative correlation between fat-free mass and leptin was observed in both groups as shown: In the BED group ( $\mathrm{r}=0.48$, $\mathrm{p}=0.04)$, in the NBED group $(\mathrm{r}=0.72, \mathrm{p}=0.005)$.

In the women who lost more than $1 \mathrm{Kg}$ over the 24-week intervention period $(n=23)$, weight reduction positively correlated with fat percentage and WC reduction, as expected and shown in Table 4. There was no correlation with biomarkers or lipids levels and binge disorder.

Table 4 Correlation analyses among leptin and anthropometric parameters: After multidisciplinary intervention

\begin{tabular}{lll}
\hline \multirow{2}{*}{ Antropometric parameters } & \multicolumn{2}{l}{ Leptin } \\
\cline { 2 - 3 } & NBED(r) & BED(r) \\
\hline BMI & $0.52^{* *}$ & $0.58^{* *}$ \\
WC & $0.56^{* *}$ & $0.60 *$ \\
Fat \% & $0.50 * *$ & $0.52 *$ \\
Fat-free mass & $-0.72 * *$ & $-0.48^{*}$
\end{tabular}

BMI, body mass index; WC, waist circumference; NBED, not binge eating disorder; BED, binge eating disorder; Pearson correlation test $*_{p}<0.05$; **p $<$ $0.01 ; * * * p<0.001$

Obese individuals with BED are one of the high-risk populations for developing MetS due to their overweight and maladaptive eating patterns. However, this unadjusted dietary pattern is not able to make its metabolic patterns different from those found in normative population studies of obese individuals without binge eating, as demonstrated by Udo et al. ${ }^{24}$ in a study of a US population. The present study confirmed that there is no interference of binge eating in metabolic alterations typical of obesity and confirmed that several anthropometric and biochemical parameters are not different when comparing obese individuals with binge eating of those without the disorder.
All the analyzed parameters were within the expected values for obese, and above those considered normal for population patterns, and were not influenced by the dietary pattern., ${ }^{719}$ Differently from our study, Succurro et al., ${ }^{25}$ demonstrated an unfavorable metabolic and inflammatory profile only in obese women with BED. They studied 115 obese individuals of both genders and divided them into 2 groups per the presence or absence of binge eating. According to the researchers, the unfavorable profile was only present in the binge eating group. These individuals had higher BMI, WC, fat mass and a lower fat-free mass when compared to non- binge eating obese as well as a poorer metabolic profile.

Leptin, a hormone derived from adipose tissue involved in the pathophysiology of eating disorders, is increased in obese women with binge eating. ${ }^{6}$ This statement was confirmed by this study: Women with BED had significantly higher levels of leptin than their counterparts without BED.

In a cross-sectional study conducted by a group of researchers at the University of Geneva, in Italy, the results were like ours. They showed that obese BED patients have higher concentrations of serum leptin than non-binging counterparts and attributed such founds to body fat size and the influence of eating behavior. ${ }^{26}$

It was observed a correlation among the level of leptin with the percentage of WC and body fat. In stationary conditions, the serum concentration of leptin is directly related to body fat reserves, being also affected by changes in the energy balance and influenced by the dietary pattern.

A small reduction in body weight of only 5 to $10 \%$, regardless of the weight before this reduction, may be sufficient for abdominal circumference, blood pressure, inflammatory cytokines, serum glucose, and triglycerides to be reduced. Weight reduction also has a significant psychological effect, improving body image, reducing anxiety and consequently binge eating. ${ }^{27}$

The prevalence of physical inactivity and hypertension was also similar between the groups, as well as the severity of obesity. ${ }^{28,29}$ After 24weeks of BT and CBT intervention, our results indicated a $4 \%$ reduction in body weight as well as a significant improvement in almost all variables analyzed regardless of the presence of binge eating disorder. Behavioral change contributed to the positive outcome of weight loss.

Satisfaction with the program was observed not only by the smile, but by a change of dress code, which has become more cheerful and jovial. There was a significant change in eating behavior measured by the BES after the intervention.

Seamoore et al., ${ }^{30}$ a research group from UK, studying BED women who attended a weekly integrative therapy group for 6 months, have previously demonstrated changes in eating behavior measured by the same Scale, after one year intervention.

Clinical, biochemical and psychological improvement through multidisciplinary treatment has been reported by other authors. ${ }^{31,32} \mathrm{~A}$ single victory would justify the intervention. However, when all these positive factors are associated, intervening in patients with BED in the short and long term becomes mandatory.

The present multidisciplinary intervention led to a significant reduction of fat mass of obese individuals with binge eating similar to the data presented by Savastano et al..$^{33} \&$ Leal et al. ${ }^{34}$ Both reports 
have shown that weight loss and reduction of binge eating have a positive effect on reducing fat mass and consequently reducing cardiovascular risk.

Although unwanted in any intervention for weight loss, a reduction in the percentage of fat-free mass associated with loss of fat mass can be expected. It should be considered that, regardless of the method used to evaluate body composition, it is possible that this component is overestimated. This can be explained by the fact that, together with the fat-free mass, there is the volume of free water, which is known to be high in obese people. ${ }^{33}$

The intervention resulted in fat-free mass reduction only in women without binge eating. This reduction may be reflecting a greater amount of free water in this group and therefore, parallel to the reduction of weight, a significant reduction of the amount of free water could have occurred, not necessarily reflecting sarcopenia. ${ }^{34}$

The multidisciplinary model of BED intervention based on personcentered care leads to greater self-confidence and self-esteem, causing changes in eating behavior and reducing compulsive episodes. ${ }^{35,36}$

Some cardiovascular risk factors as smoking habits and alcohol consumption have not changed. The short period of therapy can justify these unsuccessful results in life style change. Limitations in the present study, such as a small number of participants, the inclusion of only women, the relatively short period of intervention and different degrees of obesity may have influenced the results. Nonetheless, this study has contributed to a better understanding of BED and that multidisciplinary intervention is the key factor in treating this disorder.

Intervening in behavioral changes in obese individuals, regardless of whether there is BED, brings clinical, metabolic, psychological and emotional improvements. Investing in a person-centered, non-diseasebased approach, considering the various aspects involved in obesity and eating disorders, sensitivity and dedication to care, are key to effective and long-lasting success in treating binge eating disorder. ${ }^{37}$

\section{Conclusion}

Obese women with BED have no difference in anthropometric and biochemical parameters compared to obese without BED, except for leptin, higher in the first group. The multidisciplinary 24-week intervention improves both the anthropometric and metabolic profile and reduces episodes of binge eating.

\section{Acknowledgements}

This research project was partially funded by Rio de Janeiro Foundation Research Agency - FAPERJ and Brazilian National Council for Scientific and Technological Development -CNPq.

\section{Conflict of interest}

The author declares no conflict of interest.

\section{References}

1. Diagnostic and statistical manual of mental disorders (DSM-5®). 5th ed. USA: American Psychiatric Association; 2013. 991 p.

2. Pearl RL, White MA, Grilo CM. Overvaluation of shape and weight as a mediator between self-esteem and weight bias internalization among patients with binge eating disorder. Eat Behav. 2014;15(2):259-261.
3. Durso LE, Latner JD, White MA, et al. Internalized weight bias in obese patients with binge eating disorder: associations with eating disturbances and psychological functioning. Int J Eat Disord. 2012;45(3):423-427.

4. Kessler RC, Berglund PA, Chiu WT, et al. The prevalence and correlates of binge eating disorder in the World Health Organization World Mental Health Surveys. Biol Psychiatry. 2013;73(9):904-914.

5. Preti A, Girolamo G, Vilagut G, et al. The epidemiology of eating disorders in six European countries: results of the ESEMeD-WMH project. J Psychiatr Res. 2009;43(14):1125-1132.

6. Brandao PP, Garcia-Souza EP, Neves FA, et al. Leptin/adiponectin ratio in obese women with and without binge eating disorder. Neuro Endocrinol Lett. 2010;31(3):353-358.

7. Elks CM, Francis J. Central adiposity, systemic inflammation, and the metabolic syndrome. Curr Hypertens Rep. 2010;12(2):99-104.

8. Walther G, Obert P, Dutheil F, et al. Metabolic syndrome individuals with and without type 2 diabetes mellitus present generalized vascular dysfunction:cross-sectional study. Arterioscler Thromb Vasc Biol. 2015;35(4):1022-1029.

9. Grilo CM, Masheb RM, White MA, et al. Treatment of binge eating disorder in racially and ethnically diverse obese patients in primary care: randomized placebo-controlled clinical trial of self-help and medication. Behav Res Ther. 2014;58:1-9.

10. Ricca V, Castellini G, Lo Sauro C, et al. Correlations between binge eating and emotional eating in a sample of overweight subjects. Appetite. 2009;53(3):418-421.

11. Amianto F, Ottone L, Abbate Daga G, et al. Binge-eating disorder diagnosis and treatment: a recap in front of DSM-5. BMC Psychiatry. $2015 ; 15: 70$

12. Brambilla F, Samek L, Company M, et al. Multivariate therapeutic approach to binge-eating disorder: combined nutritional, psychological and pharmacological treatment. Int Clin Psychopharmacol. 2009;24:312-317.

13. Compare A, Calugi S, Marchesini G, et al. Emotionally focused group therapy and dietary counseling in binge eating disorder. Effect on eating disorder psychopathology and quality of life. Appetite. 2013;71:361368 .

14. Wadden TA, Webb VL, Moran CH, et al. Lifestyle modification for obesity: new developments in diet, physical activity, and behavior therapy. Circulation. 2012;125(9):1157-1170.

15. McElroy SL, Guerdjikova AI, Mori N, et al. Overview of the treatment of binge eating disorder. CNS Spectr. 2015;20(6):546-556.

16. Boutelle KN, Monreal T, Strong DR, et al. An open trial evaluating an attention bias modification program for overweight adults who binge eat. J Behav Ther Exp Psychiatry. 2016;52:138-146.

17. Physical Status: The Use and Interpretation of Anthropometry. Switzerland: World Health Organization; 1995.

18. Freitas S, Lopes C, Coutinho $\mathrm{W}$, et al. Translation and adaptation into Portuguese of the Binge-Eating Scale. Rev Bras Psiquiatr. 2001;23(4):215-220.

19. Choi KM, Kim SM, Kim YE, et al. Prevalence and cardiovascular disease risk of the metabolic syndrome using National Cholesterol Education Program and International Diabetes Federation definitions in the Korean population. Metabolism. 2007;56(4):552-558.

20. VI Brazilian Guidelines on Hypertension. Arq Bras Cardiol. 2010;95(1 Suppl):1-51. 
21. Ozhan H, Alemdar R, Caglar O, et al. Performance of bioelectrical impedance analysis in the diagnosis of metabolic syndrome. J Investig Med. 2012;60(3):587-591.

22. Ridker PM, Rifai N, Cook NR, et al. Non-HDL cholesterol, apolipoproteins A-I and B100, standard lipid measures, lipid ratios, and CRP as risk factors for cardiovascular disease in women. JAMA. 2005;294(3):326-333.

23. Godoy-Matos AF, Oliveira J, Guedes EP, et al. Diretrizes brasileiras de obesidade 2009/2010. ABESO. 2009.

24. Udo T, McKee SA, White MA, et al. The factor structure of the metabolic syndrome in obese individuals with binge eating disorder. J Psychosom Res. 2014;76(2):152-157.

25. Succurro E, Segura-Garcia C, Ruffo M, et al. Obese Patients With a Binge Eating Disorder Have an Unfavorable Metabolic and Inflammatory Profile. Medicine (Baltimore). 2015;94(52):e2098.

26. Adami GF, Campostano A, Cella F, et al. Serum leptin concentration in obese patients with binge eating disorder. Int $J$ Obes Relat Metab Disord. 2002;26(8):1125-1128.

27. Fluckiger C, Meyer A, Wampold BE, et al. Predicting premature termination within a randomized controlled trial for binge-eating patients. Behav Ther. 2011;42(4):716-725.

28. Carnier J, Sanches Pde L, da Silva PL, et al. Obese adolescents with eating disorders: analysis of metabolic and inflammatory states. Physiol Behav. 2012;105(2):175-180.

29. Masquio DC, de Piano A, Campos RM, et al. The role of multicomponent therapy in the metabolic syndrome, inflammation and cardiovascular risk in obese adolescents. Br J Nutr. 2015;113(12):1920-1930.
30. Seamoore D, Buckroyd J, Stott D. Changes in eating behaviour group therapy for women who binge eat:a pilot study. J Psychiatr Ment Health Nurs. 2006;13(3):337-346.

31. Kyle TK, Puhl RM. Putting people first in obesity. Obesity (Silver Spring). 2014;22(5):1211.

32. Matos MIR, Aranha LS, Faria AN, et al. Binge eating disorder, anxiety, depression and body image in grade III obesity patients. Rev Bras Psiquiatr. 2002;24(4):165-169.

33. Savastano S, Belfiore A, Di Somma C, et al. Validity of bioelectrical impedance analysis to estimate body composition changes after bariatric surgery in premenopausal morbidly women. Obes Surg. 2010;20(3):332-339.

34. Leal AA, Faintuch J, Morais AA, et al. Bioimpedance analysis: should it be used in morbid obesity? Am J Hum Biol. 2011;23(3):420-422.

35. Gomez-Arbelaez D, Bellido D, Castro AI, et al. Body Composition Changes After Very-Low-Calorie Ketogenic Diet in Obesity Evaluated by 3 Standardized Methods. J Clin Endocrinol Metab. 2017;102(2):488498.

36. Di Daniele N, Petramala L, Di Renzo L, et al. Body composition changes and cardiometabolic benefits of a balanced Italian Mediterranean Diet in obese patients with metabolic syndrome. Acta Diabetol. 2013;50(3):409-416.

37. Vocks S, Tuschen-Caffier B, Pietrowsky R, et al. Meta-analysis of the effectiveness of psychological and pharmacological treatments for binge eating disorder. Int J Eat Disord. 2010;43(3):205-217. 